\title{
About the Negative Gravitational Mass
}

\author{
Stéphane Le Corre \\ Ecole Polytechnique Fédérale de Lausanne, Lausanne, Switzerland \\ Email: stephane.lecorre@epfl.ch
}

How to cite this paper: Le Corre, S. (2018) About the Negative Gravitational Mass. Open Access Library Journal, 5: e4312. https://doi.org/10.4236/oalib.1104312

Received: January 4, 2018

Accepted: January 26, 2018

Published: January 29, 2018

Copyright $\odot 2018$ by author and Open Access Library Inc.

This work is licensed under the Creative Commons Attribution International License (CC BY 4.0).

http://creativecommons.org/licenses/by/4.0/

\begin{abstract}
In a previous paper, we demonstrated that the linearized general relativity could explain dark energy (the experimental values of parameters $\Omega_{\Lambda}$, the cosmological constant, the recent acceleration of the expansion of our Universe) offering an amazing image of our universe at an incredible scale. This explanation of dark energy relies on the assumption of the existence of a negative gravitational mass (with always a positive inertial mass meaning that gravitation could be repulsive). This article demonstrates that this assumption is not only compliant with general relativity but even that the repulsive gravitational interaction is inscribed in the equations of the general relativity. The absence of negative gravitational mass should then be justified because nothing forbids its existence and until now repulsive gravitation has never been observed. This natural possibility of general relativity must be then avoided by adding an ad hoc paradigm. In a way, the principle of masses' equivalence plays indirectly this role. We will show why this principle can be verified with a great accuracy but we also propose experiments that could violate this principle, allowing at the same time rejecting its status of theoretical principle. This frame of explanation (general relativity released from this ad hoc constraint) opened then the way of the negative gravitational mass with its natural corollary, the repulsive gravitational interaction, and of the following major predictions: the antimatter should have a negative gravitational mass, the neutrino should not be a Majorana particle, the principle of equivalence of masses should be violated for the antiprotonic helium, the apparent disappearance of antimatter could be explained. We recall some other consequences: an "initial" cosmic inflation would be unavoidable, dark energy (or cosmological constant) might not be constant in time (causing accelerating universe). Several experiments are testing some of these predictions: NEMO experiment tests if neutrino is a Majorana particle, and AEgIS, ALPHA and GBAR experiments at CERN test the behavior of the gravitational interaction on anti-matter and the sign of its gravitational mass. First results could be obtained in 2018. Experiments are proposed to test the violation of the principle of equivalence of the masses.
\end{abstract}




\section{Subject Areas}

Modern Physics, Theoretical Physics

\section{Keywords}

Gravitation, Gravitic Field, Negative Mass, Repulsive Gravitation, Cosmic Inflation, Dark Energy, Antimatter, Cosmological Constant, Accelerating Universe

\section{Introduction}

Certainly, the most important mystery of astrophysics is the problem of dark energy. This latter component represents around fourteen times the mass-energy of the ordinary matter. And until now, this term cannot be explained. In [1], a solution is proposed. But it imposes an assumption on gravitational mass: the gravitational mass should be negative (with always a positive inertial mass). It leads to the existence of a repulsive gravitational interaction. In the theoretical frame of [1], this assumption is deduced from the explanation of the dark energy, but the negative gravitational mass is an assumption sufficiently important to be studied directly and independently from dark energy (even if it affords a solution to that). This is the purpose of this article, testing its theoretical compliance, its rationality, with our physical theories and studying its direct logical consequences for the theory and for the experiment.

We are first going to define the linearized general relativity, from which we will justify the idea of a negative gravitational mass. As we are going to demonstrate it just after, the non-observation of a repulsive gravitation is in fact a fundamental weak point of the general relativity because this possibility is inscribed in its equations (just like anti-matter in the Dirac equations). A supplementary paradigm postulating that, negative gravitational mass is not possible, must be then erected. The principle of masses' equivalence plays indirectly this role, but is founded only on experiments. We are going to study this principle at the light of this assumption. It will allow explaining why the principle is verified with a great accuracy and at the same time how this principle can be violated. Using the quantum mechanics, we will demonstrate that antimatter must have a negative gravitational mass. And as an extremely consistent solution, it will allow explaining the apparent disappearance of the antimatter and the apparent absence of the repulsive gravitation in our Universe. We will predict some results to test all these theoretical frames for current experiments (on gravitational mass for AEgIS, ALPHA and GBAR experiments and for Majorana particle in NEMO experiment) and for new experiments (on the violation of the principle of masses' equivalence). Other consequences are also mentioned (cosmic inflation, explanation of the dark energy and recent accelerating Universe) due to this negative gravitational mass. 


\section{Negative Gravitational Mass in General Relativity}

\subsection{From General Relativity to Linearized General Relativity}

From general relativity, one deduces the linearized general relativity in the approximation of a quasi-flat Minkowski space $\left(g^{\mu \nu}=\eta^{\mu \nu}+h^{\mu \nu} ;\left|h^{\mu v}\right| \ll 1\right)$. With the following Lorentz gauge, it gives the following field equations as in [2] (with $\left.\square=\frac{1}{c^{2}} \frac{\partial^{2}}{\partial t^{2}}-\Delta\right):$

$$
\partial_{\mu} \bar{h}^{\mu v}=0 ; \quad \square \bar{h}^{\mu v}=-2 \frac{8 \pi G}{c^{4}} T^{\mu v}
$$

With:

$$
\bar{h}^{\mu v}=h^{\mu v}-\frac{1}{2} \eta^{\mu v} h ; h \equiv h_{\sigma}^{\sigma} ; h_{v}^{\mu}=\eta^{\mu \sigma} h_{\sigma v} ; \bar{h}=-h .
$$

The general solution of these equations is:

$$
\bar{h}^{\mu v}(c t, \boldsymbol{x})=-\frac{4 G}{c^{4}} \int \frac{T^{\mu v}(c t-|\boldsymbol{x}-\boldsymbol{y}|, \boldsymbol{y})}{|\boldsymbol{x}-\boldsymbol{y}|} \mathrm{d}^{3} \boldsymbol{y} .
$$

In the approximation of a source with low speed, one has:

$$
T^{00}=\rho c^{2} ; T^{0 i}=c \rho u^{i} ; T^{i j}=\rho u^{i} u^{j} .
$$

And for a stationary solution, one has:

$$
\bar{h}^{\mu v}(x)=-\frac{4 G}{c^{4}} \int \frac{T^{\mu v}(y)}{|x-y|} \mathrm{d}^{3} \boldsymbol{y} .
$$

At this step, by proximity with electromagnetism, one traditionally defines a scalar potential $\varphi$ and a vector potential $H^{i}$. There are in the literature several definitions as in [3] for the vector potential $H^{i}$. In our study, we are going to define:

$$
\bar{h}^{00}=\frac{4 \varphi}{c^{2}} ; \bar{h}^{0 i}=\frac{4 H^{i}}{c} ; \bar{h}^{i j}=0 .
$$

With gravitational scalar potential $\varphi$ and gravitational vector potential $H^{i}$ :

$$
\begin{gathered}
\varphi(\boldsymbol{x}) \equiv-G \int \frac{\rho(\boldsymbol{y})}{|\boldsymbol{x}-\boldsymbol{y}|} \mathrm{d}^{3} \boldsymbol{y} \\
H^{i}(\boldsymbol{x}) \equiv-\frac{G}{c^{2}} \int \frac{\rho(\boldsymbol{y}) u^{i}(\boldsymbol{y})}{|\boldsymbol{x}-\boldsymbol{y}|} \mathrm{d}^{3} \boldsymbol{y}=-K^{-1} \int \frac{\rho(\boldsymbol{y}) u^{i}(\boldsymbol{y})}{|\boldsymbol{x}-\boldsymbol{y}|} \mathrm{d}^{3} \boldsymbol{y} .
\end{gathered}
$$

With $K$ a new constant defined by:

$$
G K=c^{2} \text {. }
$$

This definition gives $K^{-1} \sim 7.4 \times 10^{-28}$ very small compare to $G$.

The field Equation (1) can be then written (Poisson equations):

$$
\Delta \varphi=4 \pi G \rho ; \quad \Delta H^{i}=\frac{4 \pi G}{c^{2}} \rho u^{i}=4 \pi K^{-1} \rho u^{i} .
$$

With the following definitions of $\boldsymbol{g}$ (gravity field) and $\boldsymbol{k}$ (gravitic field), 
those relations can be obtained from the following equations (also called gravitomagnetism):

$$
\begin{aligned}
& \boldsymbol{g}=-\operatorname{grad} \varphi ; \boldsymbol{k}=\operatorname{rot} \boldsymbol{H} \\
& \operatorname{rot} \boldsymbol{g}=0 ; \operatorname{div} \boldsymbol{k}=0 ; \\
& \operatorname{div} \boldsymbol{g}=-4 \pi G \rho ; \operatorname{rot} \boldsymbol{k}=-4 \pi K^{-1} \boldsymbol{j}_{p}
\end{aligned}
$$

With the Equation (2), one has:

$$
h^{00}=h^{11}=h^{22}=h^{33}=\frac{2 \varphi}{c^{2}} ; h^{0 i}=\frac{4 H^{i}}{c} ; h^{i j}=0 .
$$

The equations of geodesics in the linear approximation give:

$$
\frac{\mathrm{d}^{2} x^{i}}{\mathrm{~d} t^{2}} \sim-\frac{1}{2} c^{2} \delta^{i j} \partial_{j} h_{00}-c \delta^{i k}\left(\partial_{k} h_{0 j}-\partial_{j} h_{0 k}\right) v^{j} .
$$

It then leads to the movement equations:

$$
\frac{\mathrm{d}^{2} \boldsymbol{x}}{\mathrm{d} t^{2}} \sim-\boldsymbol{g r a d} \varphi+4 \boldsymbol{v} \wedge(\boldsymbol{r o t} \boldsymbol{H})=\boldsymbol{g}+4 \boldsymbol{v} \wedge \boldsymbol{k}
$$

\subsection{From Linearized General Relativity to the Assumption of Negative Mass and Repulsive Gravitation}

The first and the main element to introduce the negative mass is at the heart of the general relativity. As seen previously, general relativity can be linearized, leading to a rigorous simplified theory, the linearized general relativity (LGR) also known as gravitomagnetism. In term of predictions what it can be positively deduced from LGR can also be deduced from general relativity. I said "positively" in the sense that the results deduced from LGR can be less accurate or inaccessible compare to the general relativity. Fewer predictions can then be obtained from LGR than from GR but what is true for LGR is also true for general relativity. As seen at the beginning of this article, the LGR is extremely similar to the idealization of the electromagnetism. In electromagnetism, the charge can be negative. LGR also allows negative gravitational mass. One can recall that gravitational mass is not allowed because, until now, a negative gravitational mass has never been observed. But as we will see for theoretical reasons, as the electromagnetic interaction can be repulsive and attractive, the equations of general relativity are consistent with a repulsive and attractive gravitational interaction. We also show that the Newtonian gravitation, that is also a simplified theory of LGR, is compliant with negative gravitational mass.

A second element comes from the article [1], in which the assumption of a negative mass is proposed to explain dark energy. Just like to explain the dark matter far from the center of mass, where the gravity field becomes too weak, the gravitic field with a specific arrangement of the negative mass can become the main component of the gravitational interaction at very high scale. In this configuration, the gravitic field plays then the role of the cosmological constant. The negativity of the gravitational mass (associated with a positive inertial mass) allows generating a repulsive gravitational interaction as expected by the observed 
term of dark energy.

Let's consider a negative gravitational mass. A question is then what about inertial mass. Some studies show that if one considers a negative inertial mass, it leads to several inacceptable physical behaviors [4]. And certainly more important, trajectory of particles in large accelerators implies that inertial mass must be positive. By consequence, we are considering in this study that the inertial mass cannot be negative. This situation is once again very similar to electromagnetism with a charge which can be negative and an inertial mass which cannot (gravitational mass can be seen as a gravitational charge).

One can still note that some studies, ([3] [5]) for examples, have ever been published with the assumption of negative mass in general relativity. But in general, in these papers, a negative inertial mass is possible to be in agreement with the principle of equivalence of masses (we will study this principle hereafter). In our study, once again, a negative inertial mass is forbidden.

\section{Negative Mass and Repulsive Gravitation Compliant with Gravitation's Theory and Quantum Mechanics}

First, let's demonstrate that the current gravitational theories (Newtonian and general relativity) work well and are consistent with the assumption of the negative gravitational mass. For that, we need to write equations by distinguishing inertial and gravitational masses. In a second step, we will demonstrate that negative gravitational masses applied in the frame of the quantum mechanics allow predicting that the antimatter should have a negative gravitational mass.

\subsection{Negative Gravitational Mass and Newton's Laws}

With $m_{i}$ the inertial mass (always positive), $m_{g}$ the gravitational mass of the test particle and $M_{g}$ the gravitational mass of the source, the Newtonian laws are:

$$
m_{i} \frac{\mathrm{d}^{2} \boldsymbol{x}}{\mathrm{d} t^{2}}=-G \frac{m_{g} M_{g}}{r^{2}} \frac{\boldsymbol{x}}{r}=-m_{g} \nabla \varphi .
$$

With $\varphi$ the gravitational potential:

$$
\varphi(r)=-G \frac{M_{g}}{r} .
$$

These laws allow idealizing the attractive force of the gravitation between positive gravitational masses $\left(M_{g}>0\right.$ and $\left.m_{g}>0\right)$.

With the negativity of the gravitational mass, there are three others cases, $\left(M_{g}<0\right.$ and $\left.m_{g}<0\right),\left(M_{g}>0\right.$ and $\left.m_{g}<0\right)$ and $\left(M_{g}<0\right.$ and $\left.m_{g}>0\right)$. But just like in electromagnetism, these four cases generate only two kinds of behavior (the two others being symmetric), an attractive or repulsive behavior.

In the case $\left(M_{g}<0\right.$ and $\left.m_{g}<0\right)$, one has:

$$
m_{i} \frac{\mathrm{d}^{2} \boldsymbol{x}}{\mathrm{d} t^{2}}=-G \frac{\left(-\left|m_{g}\right|\right)\left(-\left|M_{g}\right|\right)}{r^{2}} \frac{\boldsymbol{x}}{r}=-G \frac{\left|m_{g}\right|\left|M_{g}\right|}{r^{2}} \frac{\boldsymbol{x}}{r}=-\left|m_{g}\right| \nabla \varphi .
$$


With $\varphi$ the gravitational potential:

$$
\varphi(r)=-G \frac{\left|M_{g}\right|}{r^{2}} .
$$

The gravitational behavior in this case is then always attractive and strictly equivalent to the precedent case.

For the two others cases $\left(M_{g}<0\right.$ and $\left.m_{g}>0\right)$ or $\left(M_{g}>0\right.$ and $\left.m_{g}<0\right)$, the Newtonian laws give:

$$
m_{i} \frac{\mathrm{d}^{2} \boldsymbol{x}}{\mathrm{d} t^{2}}=\left|m_{g}\right| \nabla \varphi
$$

These equations idealize a repulsive gravitation. The assumption of a negative gravitational mass is then consistent with the Newtonian laws, because there are no theoretical contradictions. And more than this, the negative gravitational masses extend the Newtonian laws.

\subsection{Negative Gravitational Mass and General Relativity}

By the same way that we traditionally introduce the general relativity, for instance [2], we will first define an expression for the metric component $g_{00}$ obtained from geodesics' Equation (12) and secondly we will see the consequences on the expressions of the Einstein's equations. In a third paragraph we will see that the linearized general relativity is unchanged and consistent with the negative gravitational mass.

\subsubsection{Expression of $g_{00}$}

In a weak gravitational field, one has $g_{\mu v}=\eta_{\mu v}+h_{\mu v} ;\left|h_{\mu v}\right| \ll 1$.

The equations of geodesics (12) in the Newtonian approximation give:

$$
\frac{\mathrm{d}^{2} \boldsymbol{x}}{\mathrm{d} t^{2}} \sim-\frac{1}{2} c^{2} \nabla h_{00} \text {. }
$$

From the Equation (14), one has:

$$
\frac{\mathrm{d}^{2} \boldsymbol{x}}{\mathrm{d} t^{2}}=-\frac{m_{g}}{m_{i}} \nabla \varphi
$$

One then deduces (with $\eta_{00}=1$ in agreement with this approximation):

$$
h_{00}=2 \frac{m_{g}}{m_{i}} \frac{\varphi}{c^{2}} \Rightarrow g_{00}=1+2 \frac{m_{g}}{m_{i}} \frac{\varphi}{c^{2}} \text {. }
$$

In the case $\left(M_{g}<0\right.$ and $\left.m_{g}<0\right)$, we obtain the same expression as the usual case $\left(M_{g}>0\right.$ and $\left.m_{g}>0\right)$.

We then retrieve the two previous situations for the Newtonian laws:

- $\left(M_{g}>0\right.$ and $\left.m_{g}>0\right)$ or $\left(M_{g}<0\right.$ and $\left.m_{g}<0\right)$ :

$$
g_{00}=1+2 \frac{\left|m_{g}\right|}{m_{i}} \frac{\varphi}{c^{2}} \text {. }
$$

- $\left(M_{g}>0\right.$ and $\left.m_{g}<0\right)$ or $\left(M_{g}<0\right.$ and $\left.m_{g}>0\right)$ : 


$$
g_{00}=1-2 \frac{\left|m_{g}\right|}{m_{i}} \frac{\varphi}{c^{2}} .
$$

Just like for the Newtonian laws, the last point is a situation that has never been observed (case of repulsive gravitation). Once again our assumption on negative mass can be seen as an extension of the current expression, without any inconsistency.

\subsubsection{Einstein's Equations}

The Einstein equations are:

$$
R_{\mu \nu}-\frac{1}{2} g_{\mu \nu} R=-\kappa T_{\mu \nu} .
$$

The Einstein equations can be rewritten:

$$
R_{\mu v}=-\kappa\left(T_{\mu \nu}-\frac{1}{2} g_{\mu v} T\right) .
$$

For the component $R_{00}$ and in the Newtonian approximation $\left(g_{00} \sim 1\right.$ and $T_{00}=\rho_{i} c^{2}=T$ ), one obtains:

$$
R_{00}=-\frac{1}{2} \kappa \rho_{i} c^{2} .
$$

In this Newtonian approximation, one also has $\left(g_{\mu v}=\eta_{\mu v}+h_{\mu v}\right.$ with $\left.\left|h_{\mu v}\right| \ll 1\right)$ :

$$
R_{00}=-\frac{1}{2} \nabla^{2} h_{00} .
$$

It then gives:

$$
\nabla^{2} h_{00}=\kappa \rho_{i} c^{2} .
$$

In the Newtonian approximation, the masses can be seen as constant. So, from our previous expression:

$$
g_{00}=1+2 \frac{\rho_{g}}{\rho_{i}} \frac{\varphi}{c^{2}} .
$$

General relativity gives:

$$
\nabla^{2} \varphi=\frac{\kappa}{2} \frac{\rho_{i}^{2}}{\rho_{g}} c^{4} .
$$

And from the field equations in the Newtonian gravitation:

$$
\nabla^{2} \varphi=4 \pi G \rho_{g} .
$$

One then deduces that:

$$
\kappa=\frac{8 \pi G}{c^{4}} \frac{\rho_{g}^{2}}{\rho_{i}^{2}} .
$$

The Einstein's equations are also consistent with the negative gravitational mass assumption and they are not modified by the negative gravitational mass. This result confirms the previous conclusion that the negative gravitational mass extends the domain of validity of general relativity. 
To summarize, whatever the sign of the masses, $\kappa$ is unchanged.

\subsubsection{Linearized General Relativity}

From previous results, one can deduce that the linearized general relativity is not modified. But let's demonstrate it explicitly by continuing to distinguish inertial and gravitational masses.

From our value $\kappa=\frac{8 \pi G}{c^{4}} \frac{\rho_{g}^{2}}{\rho_{i}^{2}}$ the relations (1) become:

$$
\partial_{\mu} \bar{h}^{\mu v}=0 ; \square \bar{h}^{\mu v}=-2 \frac{8 \pi G}{c^{4}} \frac{\rho_{g}^{2}}{\rho_{i}^{2}} T^{\mu v} .
$$

And now if we define (in agreement with $g_{00}$ ) the new relations (6) with $T^{\mu v}=\rho_{i} u^{\mu} u^{v}$ :

$$
\bar{h}^{00}=\frac{4 \varphi}{c^{2}} \frac{\rho_{g}}{\rho_{i}} ; \bar{h}^{0 i}=\frac{4 H^{i}}{c} \frac{\rho_{g}}{\rho_{i}} ; \bar{h}^{i j}=0 .
$$

With the same definitions of $\varphi$ and $H^{i}$ than at the beginning of our study, one obtains the new relations (9) (with $\square=\frac{1}{c^{2}} \frac{\partial^{2}}{\partial t^{2}}-\Delta$ ):

$$
\Delta \varphi=4 \pi G \rho_{g} ; \Delta H^{i}=\frac{4 \pi G}{c^{2}} \rho_{g} u^{i}=4 \pi K^{-1} \rho_{g} u^{i} .
$$

One effectively retrieves the equations of linearized general relativity. And it is the gravitational mass that appears in these equations (without use the principle of equivalence of the masses) allowing the negativity of these terms.

Once again, with the Einstein's equations in the linearized approximation, one obtains two situations:

$\left(\rho_{g}>0\right)$ : The current known situation of the gravitation's field.

$\left(\rho_{g}<0\right)$ : Gravitation's field with an opposite sign compare to the current known situation.

This last situation doesn't contradict general relativity; on the contrary it extends the range of validity of the general relativity.

One can note that with our assumption of negative gravitational mass, the linearized general relativity is completely equivalent to Maxwell idealization in term of field's equation. And it is interesting to note that LGR is the idealization of the complementary behavior compare to the electromagnetism (with attraction for masses with same sign and repulsion for masses of different sign). Maxwell and LGR define the only two possible behaviors of interaction, the two combinatory between the couple (attraction, repulsion) and the couple (same sign of the charge, opposite sign of the charge). Let's continue to use this theoretical proximity to demonstrate that if our assumption of negative gravitational mass is true, then antimatter must have a negative gravitational mass.

\subsection{Negative Gravitational Mass and Quantum Mechanics}

Previously, we have only used the gravitation theories. In this section, we will 
use quantum mechanics and its linearized version. We will demonstrate that if negative gravitational mass exists, it should be the gravitational mass of the antimatter. As one can find in literature, for example [6], general relativity seems compliant with the fact that antimatter would have, if it exists, a negative mass (symmetry of the solution in the Kerr-Newman metric). But here, by another way, because in our solution the inertial mass is always positive, we are going to demonstrate that the linearized general relativity applied in the frame of a linearized quantum mechanics leads also to the same conclusion. Because of its similarity with electromagnetism, we are going to use the same demonstration [7] than in electromagnetism (with the traditional Klein-Gordon and Dirac equations) but adapted to gravitation showing that antimatter must have a negative gravitational mass.

\subsubsection{Klein-Gordon Equation}

Let's recall how the electric charge of antimatter has been predicted. Starting with the relativistic relationship $E^{2}=\boldsymbol{p}^{2} c^{2}+m_{i}^{2} c^{4} \quad\left(m_{i}\right.$ the inertial mass), one obtains a quantum relation in Minkowski space from the momentum and energy operators:

$$
\hat{p}=\frac{\hbar}{i} \nabla \text { et } \hat{E}=i \hbar \frac{\partial}{\partial t} \Rightarrow\left(\nabla^{2}-\frac{1}{c^{2}} \frac{\partial^{2}}{\partial t^{2}}\right) \psi(t)=\frac{m_{i}^{2} c^{2}}{\hbar^{2}} \psi(t) .
$$

When we want to take into account an electromagnetic field, the evolution equation can be obtained by the following substitutions:

$$
\frac{\hbar}{i} \nabla \rightarrow \frac{\hbar}{i} \nabla-q A \text { and } i \hbar \frac{\partial}{\partial t} \rightarrow i \hbar \frac{\partial}{\partial t}-q V
$$

It is shown that a complex conjugation and a change of sign of the electric charge $q$ let invariant the wave equation (solution of the evolution Equation (34)). This "conjugated" solution can be then interpreted as the idealization of the antiparticles, associated with the particle of the same inertial mass and opposite charge.

Now, let's take into account a gravitational field. First, we show that our context of linearized general relativity is equivalent to the one of electromagnetism when the gravitational masses tend to zero, allowing applying the same previous reasoning.

In the approximation of linearized general relativity, we are in a quasi-flat Minkowski space, just like Klein-Gordon equations. More the gravitational masses (the field's source and the one that undergoes it) tend to zero, more this quasi-flat Minkowski space must tend to a Minkowski space.

With our definitions, linearized general relativity is equivalent to Maxwell idealization of electromagnetism in term of field equations, as seen in the beginning of our paper. Idealization of gravitation field equations, in this approximation, has then the same quadrivector $\left(\frac{\varphi}{c}, \boldsymbol{H}\right)$ than electromagnetism $\left(\frac{V}{c}, A\right)$. Once again more the masses tends to zero, more this approximation 
leads to this equivalence.

In the approximation of low speed, gravitational mass is invariant, just like charge in electromagnetism.

In these approximations, the only difference is in the movement Equation (13). Gravitation "applies" a constant factor " 4 " on the potential vector $\boldsymbol{H}$ (as seen in the beginning of our study).

From all these similarities, one can then deduce that in the approximation of linearized general relativity, low speed and masses tending to zero, one has, in term of movement equations, the following correspondences:

$$
\boldsymbol{A} \rightarrow 4 \boldsymbol{H} ; V \rightarrow \varphi \text { and } q \rightarrow m_{g} \text { (and same inertial mass) . }
$$

Thus, in this restricted domain of approximation, we are in a domain of validity where the idealization of gravitation can be used in our quantum mechanics' context. More the masses tend to zero, better is the validity. One can then apply our linearized general relativity's approximation to the same previous idealization with our correspondences (35) to extend KLEIN-GORDON equation to this restricted domain of gravitation. The evolution equation can be then obtained by the following substitutions:

$$
\frac{\hbar}{i} \nabla \rightarrow \frac{\hbar}{i} \nabla-m_{g} 4 \boldsymbol{H} \text { and } i \hbar \frac{\partial}{\partial t} \rightarrow i \hbar \frac{\partial}{\partial t}-m_{g} \varphi .
$$

As previously, a complex conjugation and a change of sign of the gravitational mass $m_{g}$ let invariant the equation wave. This allows showing that in the context of the existence of a negative gravitational mass, antiparticles not only have opposite electric charge but also opposite gravitational mass compared to their associated particle. One can also note that this equation would define an approximation of a quantum theory of gravitation in low speed and masses tending to zero.

\subsubsection{Dirac Equation}

We are going to see this result a little more specifically about spin $1 / 2$ particles, from the Dirac equation. The Dirac equation is an approximation of first order of the Klein-Gordon equation

$$
i \frac{\partial}{\partial t} \psi(t)=\hat{H}_{0} \psi(t)
$$

With

$$
\hat{H}_{0}=-i \alpha \cdot \nabla+m_{i} \beta
$$

And

$$
\alpha_{i}=\left(\begin{array}{cc}
0 & \sigma_{i} \\
\sigma_{i} & 0
\end{array}\right) \text { et } \beta=\left(\begin{array}{ll}
\mathbb{I} & 0 \\
0 & \mathbb{I}
\end{array}\right) \text {. }
$$

It is of dimension $4, \sigma_{i}$ representing Pauli matrices and $\mathbb{I}$ the unit matrix in dimension 2.

For a charge $q$ embedded in an electromagnetic field $(V, \boldsymbol{A})$, we have the 
Hamiltonian $\hat{H}$ :

$$
\hat{H}=\boldsymbol{\alpha} \cdot(-i \nabla-q A)+m_{i} \beta+q V .
$$

Similarly this equation can be extended to the linearized general relativity approximation (by using previous correspondences). For a mass $m_{g}$ in a gravitational field $(\varphi, \boldsymbol{H})$, we obtain (always with the factor "4" of general relativity for the movement equation):

$$
\hat{H}=\boldsymbol{\alpha} \cdot\left(-i \nabla-q \boldsymbol{A}-m_{g} 4 \boldsymbol{H}\right)+m_{i} \beta+q V+m_{g} \varphi .
$$

Traditionally in the case of the only electromagnetic field, by performing the anti-unitary processing:

$$
\psi \rightarrow C \psi=U_{C} \psi^{*} \text {. }
$$

With $U_{C}$ a unit matrix as $\beta U_{C}=-U_{C} \beta^{*}$ and $\alpha_{i} U_{C}=U_{C} \alpha_{i}^{*}$ (it is shown that we can take $U_{C}=i \beta \alpha_{2}$ ), we verify that:

$$
C \hat{H}(q) C^{-1}=-\hat{H}(-q) \text {. }
$$

It can be applied to our new Dirac equation. Taking into account the gravitational field, we obtain:

$$
C \hat{H}\left(m_{g}\right) C^{-1}=-\hat{H}\left(-m_{g}\right) .
$$

And for the two fields:

$$
C \hat{H}\left(q, m_{g}\right) C^{-1}=-\hat{H}\left(-q,-m_{g}\right) .
$$

This result shows that an antiparticle has opposite gravitational mass and charge compared to its particle with a same inertial mass.

Remarks:

In these equations, only the gravitational mass undergoes the change of sign, in agreement with the fact that the inertial mass is always positive.

From these last equations, the result was quite remarkable that antiparticles have a negative gravitational mass. Conversely, given that so far any known object is either ordinary matter or antimatter (they are two complementary states) we can deduce that all negative gravitational mass is antimatter.

One can note that in the previous demonstration, although, mathematically, one can apply the transformation $\left\{m_{g} \rightarrow-m_{g}\right\}$, physically this transformation makes sense only if one makes beforehand the assumption of the existence of negative gravitational masses. In other words, these equations don't demonstrate the existence of negative gravitational masses, but allow predicting that if such a negative mass exists, these equations imply necessarily that it is the gravitational mass of antimatter.

One can make the same remark than in the previous paragraph. The Equation (41) would define an approximation of a quantum theory of gravitation in low speed and masses tending to zero.

To conclude this paragraph, our assumption of negative gravitational mass in the frame of general relativity implies that antiparticle must have necessarily a 
negative gravitational mass (and a positive inertial mass) with exactly the same opposite value than its associated particle (just like for electric charge). So antiparticle mass cannot be a slight correction of the mass of their associated particle. It leads to the following prediction:

In our solution, there is only one possibility for antimatter gravitational mass ( $\bar{m}$ means antiparticle's mass):

$$
\bar{m}_{g}=-m_{g} .
$$

This fundamental result of our study will be soon tested as we will see it.

\section{Apparent Disappearance of Antimatter and Principle of Equivalence Explained by the Repulsive Gravitation}

At this point of our demonstration, one can say that the existence of a negative gravitational mass is clearly a natural expectation of our theories. It leads to two complementary ways of thinking. If negative gravitational mass effectively doesn't exist, it means that our theories are uncompleted because they afford this possibility. And this failure could be a way to imagine new theories. But in a complementary approach, if our theories work well, the negative gravitational mass must be able to explain its own non-observation. And if it can, this self-consistency would be a really strong point that would justify its existence. We are now going to see that effectively, miraculously, the existence of negative gravitational mass implies the apparent disappearance of antimatter (i.e. of negative gravitational mass), the apparent absence of repulsive gravitation and the apparent principle of equivalence.

\subsection{A Way Explaining the Apparent Disappearance of Antimatter and the Apparent Absence of Repulsive Gravitation}

To be completely compliant with the observations, the theory should also be consistent with the fact that, until now the repulsive gravitational interaction has never been detected. As we well know, electromagnetism favors the mix of positive and negative charges at smaller scale than ours. This particularity of this interaction leads to a phenomenon of neutralization at our scale. At the opposite, with negative gravitational masses, the repulsive gravitation between heterogeneous masses necessarily leads to a separation of the masses depending on their sign. And this effect generates accumulations of masses of the same sign. These accumulations themselves increase the range of the interaction leading to an increase of zones of homogeneous mass at high scale. It is then possible to imagine that one of these zones of homogeneous mass is our current Universe. By this way, it would explain the apparent absence of repulsive gravitation in our Universe (composed of only positive gravitational masses) because there is only a repulsive gravitation where there are at the same place a negative and a positive gravitational mass. And it also explains the apparent disappearance of antimatter because, as seen before, the antimatter must be the matter with negative gravitational mass. The antimatter should then be outside our Universe of positive gra- 
vitational masses because the repulsive gravitation cannot maintain the negative masses in a context of positive masses (and inversely).

\subsection{Negative Gravitational Mass and Principle of Equivalence}

As mentioned before, some papers have studied the assumption of the negative mass. But in general, these mentioned papers maintain the principle of equivalence. The inertial masses are then also negative. In our study, the inertial mass stays positive whatever the sign of the gravitational mass. It implies several consequences on the principle of equivalence.

\subsubsection{Extension of the Principle of Equivalence of the Masses to Be Compliant with Invariance under a Global Change of the Sign of the Gravitational Mass}

The main characteristic of an interaction depending of a charge is that the interaction can be either attractive between two charges of different sign (and repulsive between two charges of same sign) just like electromagnetism or attractive between two charges of same sign (and repulsive between two charges of different sign) just like gravitation. Therefore, this characteristic doesn't depend on the definition of the sign of all the charges. When the arbitrary choice of the sign on the first charge has been defined, all the sign of the others charges have been then defined depending on this first one. Only the first reference can be indifferently defined. In other words, the physical laws of interaction must be invariant whatever the arbitrary convention of the charges' sign, just like the physical laws must be invariant whatever the referential.

In electromagnetism, the idealization of the interaction is invariant with the change of sign of all the charges. Linearized general relativity also verifies this requirement because it is equivalent to Maxwell idealization. The theory without the principle of equivalence is then (as expected by the physical laws) invariant with the change of the sign of the gravitational mass. We could have arbitrarily chosen to define our gravitational masses negatively. Our theories of gravitation would be identical (attractive for masses of the same sign). Only the principle of equivalence is not invariant by a change of sign of the gravitational mass. In this case of change of all the signs, it would become $m_{i}=-m_{g}$. The principle of equivalence makes the gravitational interaction dependent on an arbitrary choice which poses a philosophical problem because there is no reason to arbitrarily privilege a sign. Because of this requirement, the principle of equivalence of the masses should be written in the following extended form (invariant to a change of the definition of sign):

$$
m_{i}=\left|m_{g}\right|
$$

Furthermore, we have seen that $\kappa=\frac{8 \pi G}{c^{4}} \frac{\rho_{g}^{2}}{\rho_{i}^{2}}$. And we know that current general relativity (with $\kappa=\frac{8 \pi G}{c^{4}}$ ) is verified with a great accuracy. It means that 
one must have with a great accuracy $\frac{\rho_{g}^{2}}{\rho_{i}^{2}}=1$. With our assumption of negative gravitational mass, this relation is equivalent to have $\rho_{i}=\left|\rho_{g}\right|$. By this way, for positive gravitational mass (that is the current known situation) one retrieves the same principle, meaning that the current physical results are not modified by this extension. This expression is symmetric for the choice of the sign of gravitational mass (our invariance requirement for interactions and more generally for the physical laws). This new principle is then physically relevant because if one had chosen arbitrarily negative values for all our known gravitational masses $m_{g}$ (choice as legitimate as the choice of positive gravitational masses) the physical theories would always be valid.

\subsubsection{A Way Explaining How the Principle of Equivalence Appears as a Principle}

We are now going to explain the origin of this mysterious masses' equivalence principle. This explanatory process applied to electromagnetism will also explain why the charge doesn't follow such equivalence.

Let's imagine the creation of masses by pairs of particle and antiparticle. Let's make the assumption that for each particle, one has $m_{g}=\alpha m_{i}$. A priori, the value of $\alpha$ could depend on each created particle. But let us assume that this ratio $\frac{m_{g}}{m_{i}}=\alpha$ is the same for all the initial particles. With this assumption, one can finally say that we have simply transposed our principle of equivalence to the only "first" created masses. But, first we are going to see that in our solution, gravitation implies that this principle of equivalence at the "first" created particles is automatically maintain to very large scale (until our Universe's scale). Secondly, we will propose a justification of the constancy of the initial ratio $\frac{m_{g}}{m_{i}}=\alpha \quad$ (this "first" principle of equivalence) also due to the gravitation.

As we have said previously in this study, the effect of the repulsive gravitational interaction is to "purify", to generate aggregations of homogeneous masses at large scale. And because the repulsive interaction increases the effect of homogenizing the matter with the quantity of mass, more a mass is large and more the principle will be well verified. Then, this leads naturally to maintain the ratio $\alpha$ at larger scale. Indeed, if the aggregation is composed of $N$ positive gravitational masses and $M$ negative masses, with $N \gg M$ and $M \sim 0$ (values due to the effectiveness of the repulsion with the size of the object), the gravitational mass of the object will be $M_{g}=N m_{g}-M m_{g} \sim N m_{g}$. Its inertial mass will be $M_{i}=N m_{i}+M m_{i} \sim N m_{i}$. One will have therefore $\frac{M_{g}}{M_{i}} \sim \frac{N m_{g}}{N m_{i}}=\frac{m_{g}}{m_{i}}=\alpha$. And the equality will be more accurate when the mass will be more homogeneous ("pure") whatever the mass of the object. And the homogeneity will be more important when the mass will be large. At our scale, this result is an apparent 
principle of equivalence of masses (for which we choose $\alpha=1$ ).

The problem is now to explain how the ratio can be the same for all the created pairs. Traditionally (for electromagnetism), the creation of the pairs is idealized as a phase's transition. We are in the same situation for gravitation. A phase's transition is characterized by a set of well-defined values of parameters. In other words, each creation of pair is made in one specific physical context. It is quite natural to expect that at a specific physical context one has a specific physical response. In our case, it could mean that the ratio $\frac{m_{g}}{m_{i}}=\alpha$ could be relatively constant. Furthermore in our solution, an initial inflation is unavoidable ([1]) because of the repulsion between the particle and antiparticle of a pair. It means that at this step, the extension area, which will become our Universe, is very small, making the constancy of $\alpha$ on this area more probable.

One can try to apply the same procedure to define the values of electrical charges, with the difference that the ratio $\alpha=\frac{q}{m_{i}}$ has then a physical unit. But this time, the electromagnetic interaction is attractive for opposite charges. It will tend to create, at "large" scale, neutral objects or charges either slightly positive or negative (slightly compare to the number of charged particles that composed the object), but unrelated to the ratio $\alpha$. Indeed, if the object is composed of $N$ positive electrical charges and $M$ negative charges, with $M \sim N$ (values due to the effectiveness of the electromagnetic attraction to create neutral objects), the charge of the final object will be then $Q=(N-M) q$. Its inertial mass will be $M_{i}=(N+M) m_{i}$. One will have therefore $\frac{Q}{M_{i}}=\frac{(N-M)}{(N+M)} \alpha \neq \alpha$. Furthermore, at the apparition of electromagnetism, the inflation decreases implying that even $\alpha$ is less constant than for gravitation. At our scale, the result is no principle of equivalence between the electric charge and the inertial mass.

\subsubsection{A Way Explaining Why the Principle of Equivalence Are Not Strictly a Principle}

We have just seen that gravitation interaction increases its capacity of purification with "time" (more precisely with the accumulation of masses). It leads to a situation where the masses equivalence principle is verified with greater and greater accuracy, tending more close to one of the two asymptotic situations (in our Universe, it is the first situation that happens):

- An object composed of only positive gravitational masses tending to $m_{g}=m_{i}$.

- An object composed of only negative gravitational masses tending to $m_{g}=-m_{i}$.

To be able to violate the principle with a measurable discrepancy, we have to generate some matter composed with particles and antiparticles in a similar proportion. And to avoid the problem of increasing the efficiency of homogenizing of the gravitation, we have to test it on matter at smaller scale than ours 
(to maintain more easily the cohabitation of matter and antimatter). Nowadays, we are able to create antiprotonic helium. In our solution, this element would be a very good candidate to violate the principle of equivalence of masses because it is composed of two protons, one or two neutrons, one electron and one antiproton, which ensures a high mixing rate. We will treat quantitatively the violation of the principle for this element in the next section. But one can also note that, generally, the experiments that test the principle of equivalence use objects with large masses which, in this theoretical frame, increase the validity of the equivalence and by the same time does not facilitate its violation.

\section{Experiments Testing Previous Predictions}

\subsection{Testing Negative Gravitational Mass of Antimatter}

There are some experiments at CERN that study the behavior of antimatter in a gravitational field (AEgIS, ALPHA, GBAR experiments). From our previous study, one can then deduces that, in such experiments, only one experimental result on gravitational mass is compliant with our solution $\bar{m}_{g}=-m_{g}$ (46). The first results should be obtained this year 2018 .

\subsection{Testing Majorana Particles}

Experiments on the search of Majorana particles are also impacted by our solution, for example NEMO Experiment. A Majorana particle is a fermion that is its own antiparticle. But with the negative gravitational mass of antiparticles, in our solution, an antiparticle with a not null gravitational mass is always different from its particle. One then cannot have a Majorana particle. It leads to another prediction:

The observation of neutrinoless double beta decay would contradict our solution. And more generally, no particle with a not null gravitational mass can be a Majorana particle (i.e. a particle that is its own antiparticle).

\subsection{Violation of the Principle of Equivalence}

The extended principle (47) and its possible violation are certainly ones of the most disturbing results of our study. A thing is sure, with our current knowledge, only experiments can validate or invalidate these predictions. And fortunately, as said before, we made a prediction that can soon test the validity of the assumption of a negative gravitational mass. And because of our knowledge on the trajectories of antiparticles obtained in the accelerators of particles, if negative gravitational mass is discovered, it will be impossible to maintain the same principle of equivalence (the particles' trajectories are consistent with $\frac{q}{m_{i}}$ with only $m_{i} \geq 0$ ). The discovery of a negative gravitational mass would then lead ineluctably to the violation of this principle that would be required in the frame of this solution. A direct experiment on the violation of the principle can be imagined. When we are in our positive gravitational universe, the principle of equivalence 
of masses is verified with a great accuracy (and it should be greater if the mass is greater). But with our assumption of negative gravitational mass this equivalence cannot be always strictly verified. From our study, if an object is a mixing of matter and antimatter, the equivalence of masses is in fact always strictly violated. This failure of the masses' equivalence should then be experimentally measurable for very simple elements that mix matter and antimatter with similar quantities. For example, antiprotonic helium should clearly show a great difference between inertial mass and gravitational mass. Our solution predicts the following violation of equivalence of masses for the antiprotonic helium (with $m_{i p}$ and $m_{g p}$, the inertial and gravitational masses of the proton and neutron, and $m_{i e}$ and $m_{g e}$, the inertial and gravitational masses of the electron):

$$
\begin{aligned}
& \bar{p}^{3} \mathrm{He}^{+}: \\
& m_{i}=3 m_{i p}+1 m_{i e}+1 \bar{m}_{i p} \sim 3 m_{i p}+1 m_{i p} \sim 4 m_{i p} \\
& m_{g}=3 m_{g p}+1 m_{g e}+1 \bar{m}_{g p} \sim 3 m_{g p}-1 m_{g p} \sim 2 m_{g p} . \\
& \bar{p}^{4} \mathrm{He}^{+}: \\
& m_{i}=4 m_{i p}+1 m_{i e}+1 \bar{m}_{i p} \sim 4 m_{i p}+1 m_{i p} \sim 5 m_{i p} \\
& m_{g}=4 m_{g p}+1 m_{g e}+1 \bar{m}_{g p} \sim 4 m_{g p}-1 m_{g p} \sim 3 m_{g p} .
\end{aligned}
$$

So, if we suppose that $m_{g p}=m_{i p}$ (i.e. that the proton and neutron are "pure" objects), one can deduce that $m_{i} \sim 2 m_{g}$ for antiprotonic helium $\bar{p}^{3} \mathrm{He}^{+}$.

\section{Discussion}

In this paper, we deduced several direct consequences of the assumption of the negative gravitational mass with always a positive inertial mass. Most of these consequences allow going further to imagine possible indirect uses or tests and new hypothetical theoretical ideas.

The extended principle of equivalence and its potential violation (presented in Section 4.2.3) could be a way of testing the elementary characteristic of a "fundamental" particle. In our explanation, more an object is pure (composed of only one kind of matter, positive or negative), more the principle will be verified. In other words, if the principle is violated the tested object is then composed of matter and antimatter. And the discrepancy will be a measure of the rate of the mixing. A minimal test for a particle to be elementary would be to not violate the principle of equivalence. But if the principle is verified, this condition would unfortunately not sufficient to ensure that the particle is elementary.

The demonstration of the propagation of the principle at large scale (presented in Section 4.2.2) illustrates a mechanism that uses a parameter $\alpha$. To be a well-founded mechanism, this parameter $\alpha$ must become a fundamental parameter that should be explained by a theory. It could be an approach to think about a more fundamental theory (and why not a unified theory if this mechanism could be applied to a similar parameter in the electromagnetism context).

One of the reasons why we have proposed the assumption of the negative mass is the similarity of the linearized general relativity and the Maxwell's equa- 
tions of electromagnetism (presented in Section 2.1). In electromagnetism, the Maxwell's equations lead to the conservation of the electric charge. This similarity allows imagining another audacious assumption, that gravitational mass could be a relativistic invariant (like the electric charge), unlike the inertial mass, leading to a kind of hypothetical relation $m_{i}=\gamma m_{i 0}=\gamma\left|m_{g}\right|$. This relation could be tested by another kind of violation of the principle of equivalence depending on the speed of the mass.

This paper is an extraction of the paper [1] that treats the problem of the dark energy. In [1], other interesting consequences of the assumption of the negative mass are presented that have not been developed in the current paper. This assumption would imply a cosmic inflation and could explain the dark energy, variable with time, causing the recent acceleration of our Universe. As seen before, in this frame of explanation, the antiparticles would not have disappeared, but they would be beyond our Universe of positive gravitational masses. And (as presented in Section 4.1) it then leads naturally to think that universes of positive masses and universes of negative masses should exist. At larger scale than our Universe, these universes would be like "fundamental" particles. At this very large scale, the principle of equivalence should be once again violated, by the mixing of negative and positive masses, just like at the very small scale of the antiprotonic helium. If this explanation were correct, the dark energy would be our first direct measure of the influence of the negative gravitational mass and by the same time our first direct measure of the influence of these universes that would surround our own Universe. It is a breathtaking picture.

\section{Conclusions}

This paper studies the assumption of the negative gravitational mass (with always positive inertial mass). It demonstrates that the Newtonian laws and the general relativity are consistent with it (and even that its possibility is inscribed in the equations of the general relativity), giving birth to a repulsive gravitation, leading to the two situations:

- Attractive gravitation: $\left(M_{g}>0\right.$ and $\left.m_{g}>0\right)$ or $\left(M_{g}<0\right.$ and $\left.m_{g}<0\right)$.

- Repulsive gravitation: $\left(M_{g}>0\right.$ and $\left.m_{g}<0\right)$ or $\left(M_{g}<0\right.$ and $\left.m_{g}>0\right)$.

In fact a third situation (on the null masses) can be imagined that is not studied in this paper, but developed in [1] that inspired this paper.

Integrated this assumption in quantum mechanics, we deduce that antiparticles should have a negative gravitational mass. And right now there are some experiments that can test this theoretical frame. There are experiments (AEgIS, ALPHA and GBAR) at CERN that will give soon results on the gravitation of antiparticles. The solution presented here implies that antiparticle has exactly the opposite gravitational mass than its associated particle $\left(\bar{m}_{g}=-m_{g}\right.$ (46)). Our solution also implies that NEMO experiment should not find evidence for the neutrinoless double beta decay. More generally, particle with a not null gravitational mass could not be a Majorana particle. We noted that the repulsive gravi- 
tation could then explain the apparent disappearance of antimatter (rejected far from our Universe of positive gravitational masses due to the negative gravitational mass of antimatter) thanks to its power of homogenization and at the same time the apparent absence of a repulsive gravitation (only possible with a mix of particle and antiparticle). This gravitational behavior is the complementary behavior of the electromagnetism for which the mix of positive and negative particles is privileged.

We demonstrate why the principle of masses' equivalence is verified with a great accuracy, but showing that it is not strictly a principle because it should be violated for a mixing of particles and antiparticles. To confirm these explanations, we predict that the antiprotonic helium $\bar{p}^{3} \mathrm{He}^{+}$violates the principle with $m_{i} \sim 2 m_{g}$.

With this assumption on gravitational mass, gravitation leads to a more extraordinary cosmology than with only gravitic field, with a change of scale. Our own Universe could be just one little zone of positive gravitational mass lost in a set of universes and anti-universes. These anti-universes should follow the same physical law and give rise to the same symmetrical objects, anti-atoms (anti-Hydrogen...), anti-molecules (anti-water...), anti-star... and why not anti-biology. At very large scale, a universe might look like a "particle" with a positive or negative gravitational mass, a cluster of universe like sets of particles... A cosmic inflation would also be unavoidable. And, in this cluster of universes and anti-universes, interaction between these universes seems to be able to explain dark energy and the recent acceleration of the expansion of our Universe.

To end, one can recall that the principle of equivalence of the masses is one the main inspirations that has given birth to the general relativity. Someone could say that, ironically, these equations allow affirming that this principle is not a principle. But fortunately, the general relativity allows by the same time understanding why the equivalence of the masses can be verified with such an accuracy. The logic is preserved.

\section{References}

[1] Le Corre, S. (2015) Dark Energy, a New Proof of the Predictive Power of General Relativity. https://hal-ens-lyon.archives-ouvertes.fr/ensl-01122689

[2] Hobson, M., et al. (2006) General Relativity.

[3] Benoit-Levy, A. (2009) Study of the Concordance of a Matter-Antimatter Symmetric Dirac-Milne Universe. https://tel.archives-ouvertes.fr/tel-00442948/

[4] Bondi, H. (1957) Negative Mass in General Relativity. Reviews of Modern Physics, 29, 423-428. https://doi.org/10.1103/RevModPhys.29.423

[5] Ni, G.-J. (2003) A New Insight into the Negative-Mass Paradox of Gravity and the Accelerating Universe. Fudan University, Shanghai. https://arxiv.org/abs/physics/0308038

[6] Chardin, G. (1997) Hyperfine Interactions.

[7] Rouge, A. (1997) Introduction to Subatomic Physics. 